\title{
Debye-scale solitary structures measured in a beam-plasma laboratory experiment
}

\author{
B. Lefebvre ${ }^{1}$, L.-J. Chen ${ }^{1}$, W. Gekelman ${ }^{2}$, P. Kintner ${ }^{3}$, J. Pickett ${ }^{4}$, P. Pribyl ${ }^{2}$, and S. Vincena ${ }^{2}$ \\ ${ }^{1}$ Space Science Center, University of New Hampshire, Durham, NH 03824, USA \\ ${ }^{2}$ Basic Plasma Science Facility, University of California, Los Angeles, CA 90095, USA \\ ${ }^{3}$ School of Electrical and Computer Engineering, Cornell University, Ithaca, NY 14853, USA \\ ${ }^{4}$ Department of Physics and Astronomy, University of Iowa, Iowa City, IA 52242, USA
}

Received: 6 August 2010 - Revised: 20 December 2010 - Accepted: 10 January 2011 - Published: 25 January 2011

\begin{abstract}
Solitary electrostatic pulses have been observed in numerous places of the magnetosphere such as the vicinity of reconnection current sheets, shocks or auroral current systems, and are often thought to be generated by energetic electron beams. We present results of a series of experiments conducted at the UCLA large plasma device (LAPD) where a suprathermal electron beam was injected parallel to a static magnetic field. Micro-probes with tips smaller than a Debye length enabled the detection of solitary pulses with positive electric potential and half-widths 4-25 Debye lengths $\left(\lambda_{D e}\right)$, over a set of experiments with various beam energies, plasma densities and magnetic field strengths. The shape, scales and amplitudes of the structures are similar to those observed in space, and consistent with electron holes. The dependance of these properties on the experimental parameters is shown. The velocities of the solitary structures (1-3 background electron thermal velocities) are found to be much lower than the beam velocities, suggesting an excitation mechanism driven by parallel currents associated to the electron beam.
\end{abstract}

\section{Introduction}

Debye-scale electrostatic solitary structures with dipolar electric fields have been abundantly observed in various boundary layers and regions supporting large-scale current systems or intensive energy dissipation. Examples include the auroral acceleration region (Temerin et al., 1982), the plasma sheet boundary layer (Matsumoto et al., 1994) and polar cap boundary layer (Tsurutani et al., 1998), the vicinity of magnetic reconnection $\mathrm{X}$-lines at the magnetopause

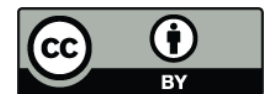

Correspondence to: B. Lefebvre (bertrand.lefebvre@unh.edu)
(Cattell et al., 2002; Matsumoto et al., 2003) or in the magnetotail (Cattell et al., 2005), the magnetosheath (Pickett et al., 2003) or the quasi-perpendicular Earth bow shock (Bale et al., 2002). They have also been observed in a laboratory magnetic reconnection experiment where they were statistically found to be associated with large inductive electric fields and energetic electron bursts (Fox et al., 2008). Kinetic numerical simulations of guidefield magnetic reconnection have also shown the generation of this type of solitary structures, interpreted as electron phase-space holes (see e.g. Schamel, 2000 for a review), in association with energetic electron populations (Drake et al., 2003; Pritchett, 2005; Goldman et al., 2008). Their generation mechanism and what determines their properties remain however in some cases not fully understood, nor is the role they might play in particle scattering and energy dissipation.

To our knowledge the only laboratory experiments dedicated to electron holes were conducted in a strongly magnetized Q-machine (Saeki et al., 1979; Lynov et al., 1979; see also Guio et al., 2003 for a review). The holes were generated by an externally applied voltage pulse and propagated into a plasma column 3-10 Debye lengths $\left(\lambda_{\mathrm{De}}\right)$ in diameter, severely limiting their perpendicular extension. These experiments were able to reproduce electron hole properties discovered in early 1-D electrostatic PIC simulations (Roberts and Berk, 1967; Berk et al., 1970), such as propagation over long distances (a few hundreds of $\lambda_{\mathrm{De}}$ ) and occasional coalescence. At the time of these experiments it was known that 2- and 3-D electron holes are unstable in unmagnetized plasmas (Morse and Nielson, 1969), but it was later found that a magnetic field allows the existence of long-lived electron hole-like structures in higher dimensions (Miyake et al., 1998; Muschietti et al., 1999; Oppenheim et al., 1999).

Published by Copernicus Publications on behalf of the European Geosciences Union and the American Geophysical Union. 
In this paper we report observations of Debye-scale solitary structures generated by injection of a supra-thermal electron beam in a laboratory experiment at UCLA's Large Plasma Device (LAPD). The purpose of this experiment is to study in a controlled environment the properties of beamgenerated solitary structures unconstrained by the size of the plasma chamber.

\section{Experimental setup}

The experiment was conducted at the upgraded Large Plasma Device (LAPD) (Gekelman et al., 1991), a helium plasma column $17.1 \mathrm{~m}$ long with a $60 \mathrm{~cm}$ diameter. The discharge was pulsed at $1 \mathrm{~Hz}$ and pulses lasted several milliseconds. In our experiments the plasma density ranged from 1.1 to $5.7 \times$ $10^{9} \mathrm{~cm}^{-3}$ and the plasma was permeated by a static magnetic field parallel to the column axis whose intensity was set from 100 to $750 \mathrm{G}$. An electron beam from 0.4 to $1 \mathrm{~cm}$ in diameter was injected for $140 \mu$ s in the direction parallel to the magnetic field. The beam density $5 \mathrm{~cm}$ from the source was found to be approximately $25 \%$ of the background electron density. Floating potentials are measured at $20 \mathrm{GHz}$ by a micro-probe (Chiang, 2010) with $10 \mu \mathrm{m}$ wide tips separated by 40 to $130 \mu \mathrm{m}$. The probe tips were aligned with the magnetic field, allowing estimation of the parallel electric field. The distance between tips is comparable to the Debye length. The probe was located on the column central axis, 6-24 cm away from the beam source. Therefore in this experiment only a small fraction of the plasma chamber was used, far away from any boundary. The electron mean-free path ranged from 11 to $42 \mathrm{~cm}$, and therefore at these scales the plasma can be considered essentially collisionless. See Lefebvre et al. (2010) for more details.

\section{Solitary wave measurements}

A $0.3 \mu$ s long time series of electric potential and field containing isolated structures is shown in Fig. 1. The solitary structure time-scale is typically one to three plasma periods $f_{\mathrm{pe}}^{-1}\left(f_{\mathrm{pe}}^{-1}=2 \mathrm{~ns}\right.$ in this case $)$, with a corresponding broadband frequency signature ranging from roughly $f_{\text {pe }} / 10$ to slightly above $f_{\text {pe }}$. A lower-frequency wave with frequency about $0.06 f_{\mathrm{pe}}$ or $5.3 f_{\mathrm{lh}}$ (where $f_{\mathrm{lh}}=$ $\left(f_{\text {ce }} f_{\text {ci }} /\left(1+f_{\text {ce }}^{2} / f_{\text {pe }}^{2}\right)\right)^{1 / 2}$ is the lower-hybrid frequency) is also simultaneously observed. The time-series typically contain a mixture of waves, localized wavepackets and solitary structures which range from the lowest frequencies in the amplifier's range $\left(5-10 f_{\mathrm{lh}}\right)$ to above the electron plasma frequency.

A solitary structure is shown in more details on Fig. 2. In this experiment the beam voltage was set to $90 \mathrm{~V}$, the background plasma density to $3.5 \times 10^{9} \mathrm{~cm}^{-3}$ and the magnetic field to $600 \mathrm{G}$, corresponding to a ratio of electron cyclotron to plasma frequencies $f_{\text {ce }} / f_{\text {pe }}=3.2$. The structure
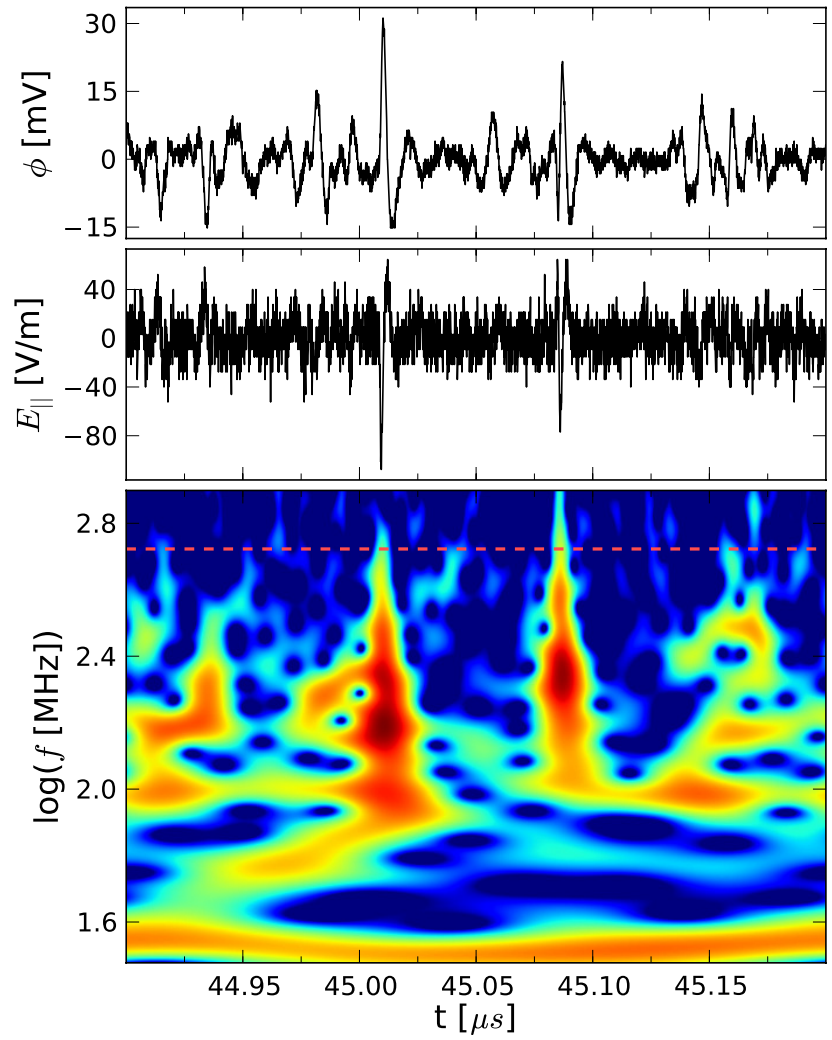

Fig. 1. Potential (top panel) and electric field (middle) displaying isolated structures and electric field wavelet spectrum (bottom). The horizontal dashed on the lower panel shows the electron plasma frequency. Wavelet coefficients are shown on a logarithmic scale.

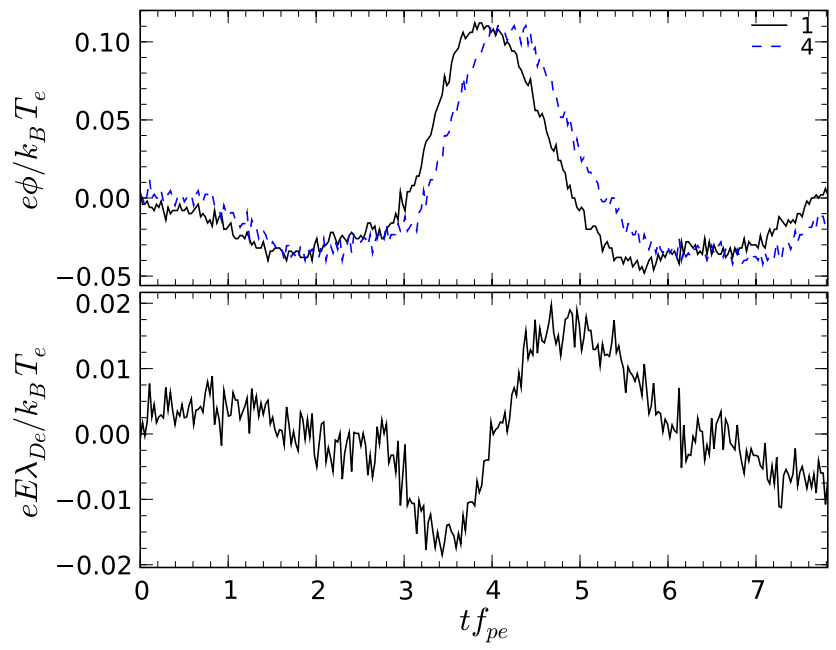

Fig. 2. A solitary positive hump with corresponding dipolar parallel electric field measured on the two most distant probe tips. Here and elsewhere, the parameters used for normalization correspond to the background electron populations prior to the beam injection. 


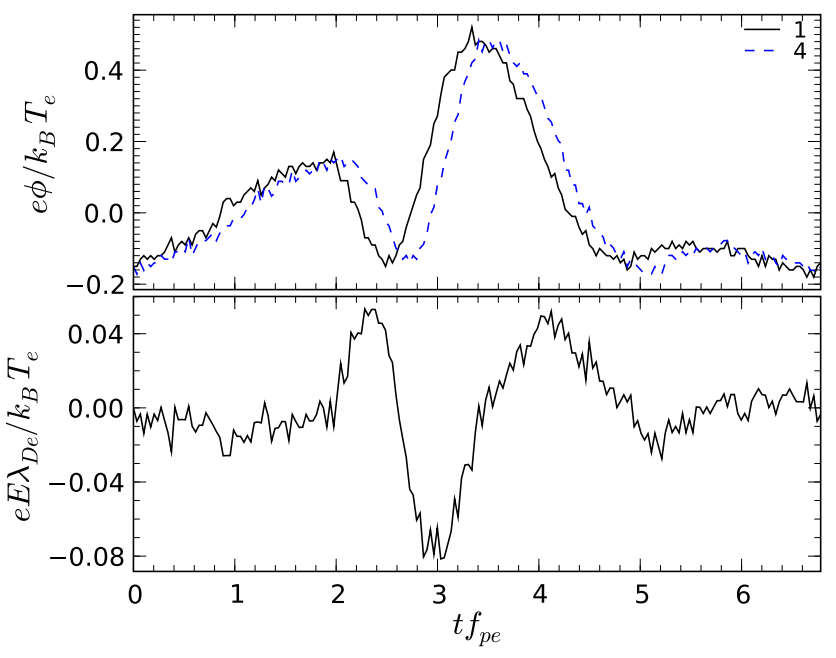

Fig. 3. A structure with a tripolar electric field.

consists of an isolated positive potential hump reaching $10 \%$ of the background electron thermal energy and a dipolar electric field. The potential hump has a very similar profile on probe tips separated by $2.3 \lambda$ De. From the time-delay between the tips the structure parallel velocity is estimated to be $v_{\|}=1.5 v_{\mathrm{Te}}$, where $v_{\mathrm{Te}}$ is the thermal velocity of the background electrons, and its parallel half-width is $L_{\|}=$ $7 \lambda_{\text {De }}$. The shape and properties of this potential pulse are consistent with an electron hole. The structure velocity is much smaller than the beam velocity, $v_{\mathrm{b}} \sim 28 v_{\mathrm{Te}}$.

While the majority of the structures have a shape similar to the one just described, some differ. Approximately $30 \%$ of the structures have an electric field which is predominantly tripolar. An example is shown on Fig. 3. In this experiment the beam voltage is $120 \mathrm{~V}$, the background plasma density is $5.7 \times 10^{9} \mathrm{~cm}^{-3}$ and the magnetic field is $750 \mathrm{G}\left(f_{\mathrm{ce}} / f_{\mathrm{pe}}=\right.$ $3)$. The structure displays a negative potential dip down to $-0.2 T_{\mathrm{e}}$ followed by a positive one reaching $0.4 T_{\mathrm{e}}$. Across the region of large electric fields a net potential drop of about $0.2 T_{\mathrm{e}}$ is observed. From the time delay between probe tips one finds $v_{\|}=2.6 v_{\mathrm{Te}}$ and a half-width for the positive potential hump $L_{\|}=15 \lambda_{\text {De }}$. The scale, velocity and amplitude of the structure are therefore larger but comparable to the previous one which suggests they could be of a similar nature. However the short delay between the probe tips do not allow to find out if this structure conserves it shapes as it propagates or whether it is only transitory. Nevertheless the tripolar electric field and potential drop are reminiscent of observations in the auroral region (Temerin et al., 1982; Bostrom et al., 1988; Pickett et al., 2004b) or the solar wind (Mangeney et al., 1999) which were interpreted as weak double layers. Tripolar structures have also been interpreted in terms of nested ion and electron holes (Pottelette and Treumann, 2005), although the estimated velocity of the structure is here probably too large for such an interpretation.
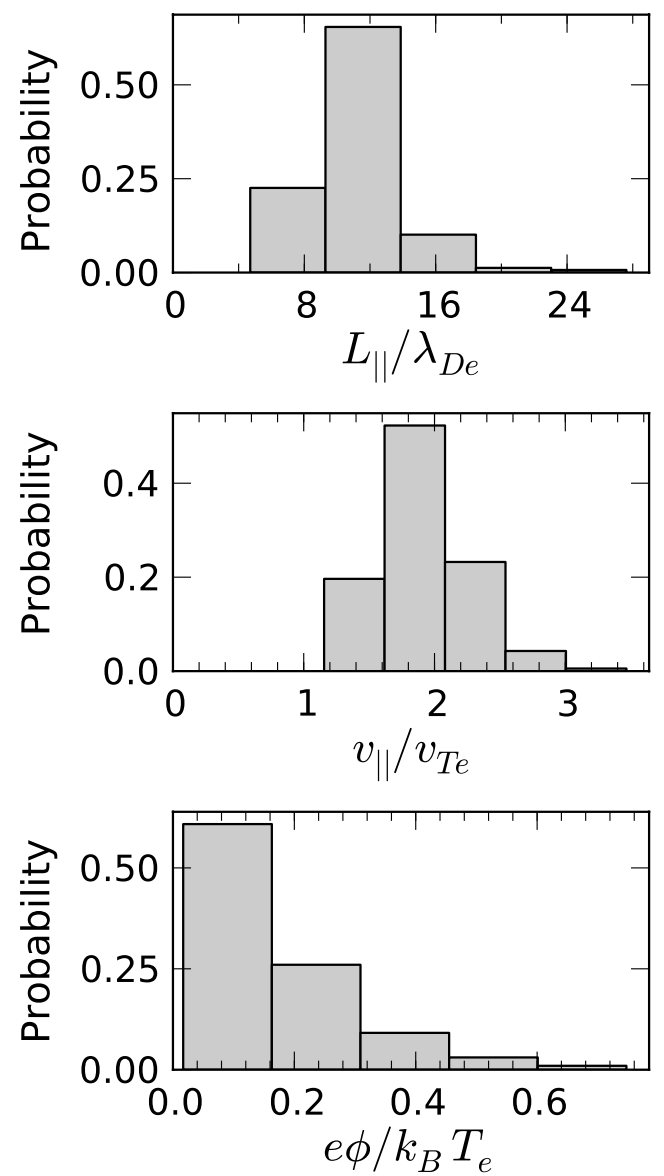

Fig. 4. Histograms of the probability of occurrence of the structure parallel half-widths (top), parallel velocities (middle) and electric potential amplitude (bottom).

\section{Statistical properties}

Times-series from 35 experiments with different magnetic fields, plasma densities or beam voltages were searched for isolated structures with positive potential humps exceeding three times the local standard deviation. Only waveforms displaying a high degree of correlation between the probe tips were considered, leaving a total of 363 unique structures. The propagation time from one probe to another was determined by cross-correlation analysis.

Histograms of the structure amplitudes, velocities and half-width are shown on Fig. 4. The median electric potential amplitude is $0.13 T_{\mathrm{e}} / e . \quad L_{\|}$ranges from 4.5 to $24.4 \lambda_{\mathrm{De}}$ with a median of $10.2 \lambda_{\text {De. }} 80 \%$ of the structures have velocities between 1.3 and $2.3 v_{\mathrm{Te}}$, with a median of $1.8 v_{\mathrm{Te}}$. These parameters are comparable to those of the solitary structures typically observed in space. Ergun et al. (1998) report observations in the auroral regions with an average half-width of $1.8 \lambda_{\mathrm{De}}$, and no half-width larger than $8 \lambda_{\mathrm{De}}$, amplitudes up to $1.2 T_{\mathrm{e}} / e$ and velocities less than $2 v_{\mathrm{Te}}$. In the cusp and the plasma sheet, Franz et al. (2005) have 


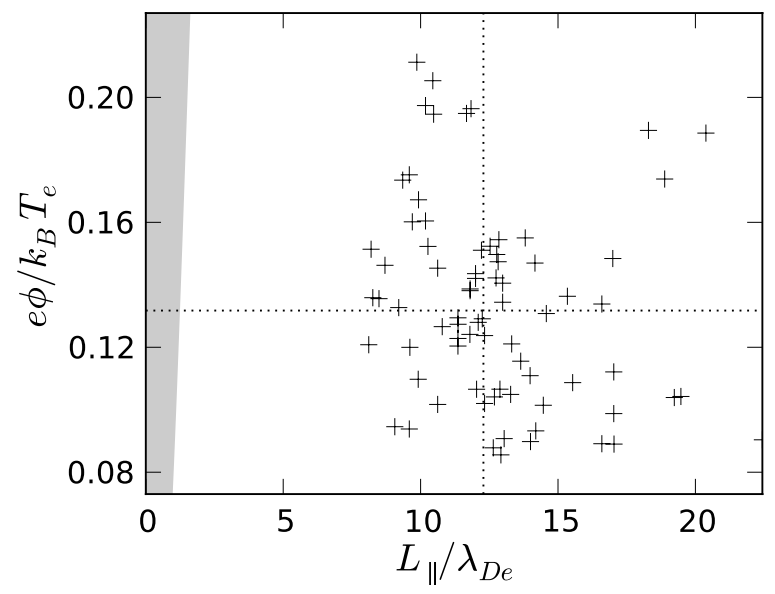

Fig. 5. Amplitude and half parallel width of the solitary structures from a single experiment, with $B=750 \mathrm{G}, n_{\mathrm{e}}=5.8 \times 10^{9} \mathrm{~cm}^{-3}$, $T_{\mathrm{e}}=0.2 \mathrm{eV}$ and a beam voltage of $120 \mathrm{~V}$. The shaded area corresponds to a theoretically inaccessible area of the parameter space for stationary 3-D electron-holes (Chen et al., 2005). The dotted lines indicate the median values.

found half-width ranging from 0.2 to $40 \lambda_{\mathrm{De}}$, velocities from 0.05 to $2 v_{\mathrm{Te}}$ and amplitudes from $3 \times 10^{-4}$ to $0.3 T_{\mathrm{e}} / e$. Recently, even larger solitary structures of half-width 10 to $70 \lambda_{\mathrm{De}}$, amplitudes $\sim 0.5 T_{\mathrm{e}} / e$ and with a finite parallel magnetic field perturbation were found in the plasma sheet (Andersson et al., 2009). The solitary structures measured in at the Versatile Toroidal Facility reconnection experiment typically had a velocity $1.5 v_{\mathrm{Te}}$ and half-width of $30 \lambda_{\mathrm{De}}$. The $1-\mathrm{D}$ electron holes in the laboratory experiments by Saeki et al. (1979) and Lynov et al. (1979) had half-widths 7$10 \lambda_{\mathrm{De}}$ and amplitudes up to $1.5 T_{\mathrm{e}} / e$. The structures we observe therefore fill the gap in size between the smaller and larger observed structures. Their amplitudes are fairly average, and their velocities are probably a bit larger than the average but nevertheless comparable to previous space observations. Let us also note a potential normalization issue when comparing our results to previous observations, as our results are normalized with respect to the temperature of the background electron population and velocities would be a bit smaller when normalized with respect to the total electron temperature.

Certain nonlinear waves such as the soliton solutions of the Kortweg-de Vries equation have a one-to-one relationship between their width and amplitude. This equation can be used to describe nonlinear electrostatic fluctuations in the small-amplitude limit, and predicts solitons whose amplitudes are a decreasing function of their widths. Figure 5 shows a scatter plot of the structure amplitudes and parallel half-width for a single experiment. In this case, $B=750 \mathrm{G}$, $n_{\mathrm{e}}=5.8 \times 10^{9} \mathrm{~cm}^{-3}, T_{\mathrm{e}}=0.2 \mathrm{eV}$ and the beam voltage is $120 \mathrm{~V}$. Within the limited range of amplitudes and widths of these structures, no significant relationship can be found. Since all the structures correspond to a single experiment

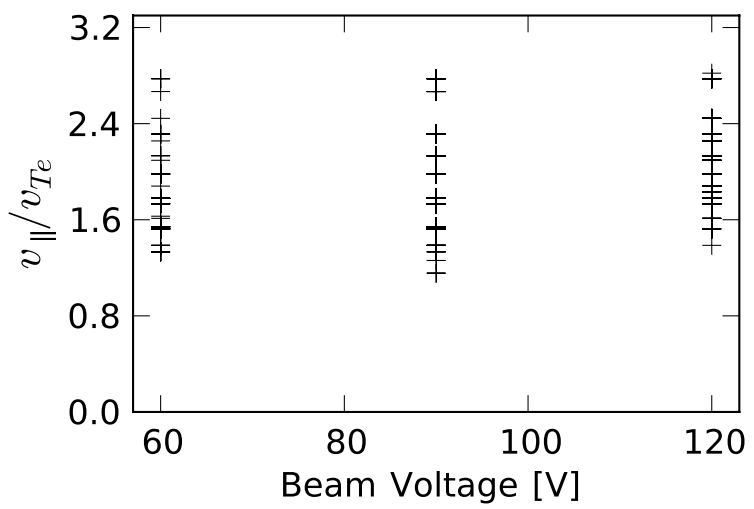

Fig. 6. Parallel velocity of the structures for various beam voltages.

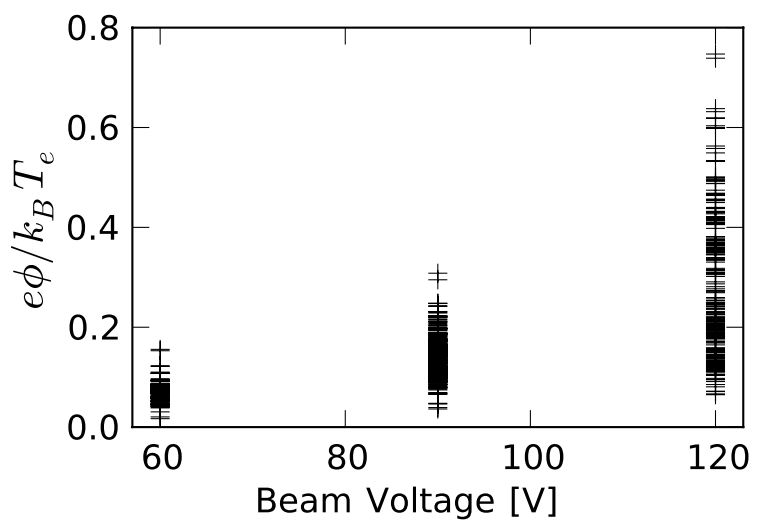

Fig. 7. Structure amplitudes (electric potential) for various beam voltages.

with a given set of parameters, the variety of sizes and amplitudes cannot be attributed to a variability in plasma parameters. No relationship was found either when plotting amplitude versus width over the whole set of experiments (Lefebvre et al., 2010). Using the constraint that the trapped electron distribution function must remain positive, Chen et al. (2005) have derived an amplitude-width inequality for stationary electron-holes in strongly magnetized plasmas which provides an upper limit to the amplitude for a given width. This upper limit increases with the parallel width. Figure 5 shows that all of the solitary pulses we observe are well within the allowed parameter space for electron-holes.

Investigating how the solitary structure properties vary as a function of the experiment parameters might provide further clues on their nature and generation mechanism. A plot of the velocities for various beam voltages is shown on Fig. 6. The beam voltage is a proxy for the beam energy, which is typically about $10 \%$ smaller. Within the range investigated (representing a factor two in beam energy) the velocities do not exhibit any noticeable dependence on the beam voltage. This observation combined with the fact that the velocities are much lower than the beam velocities excludes a generation mechanism by a resonant instability 


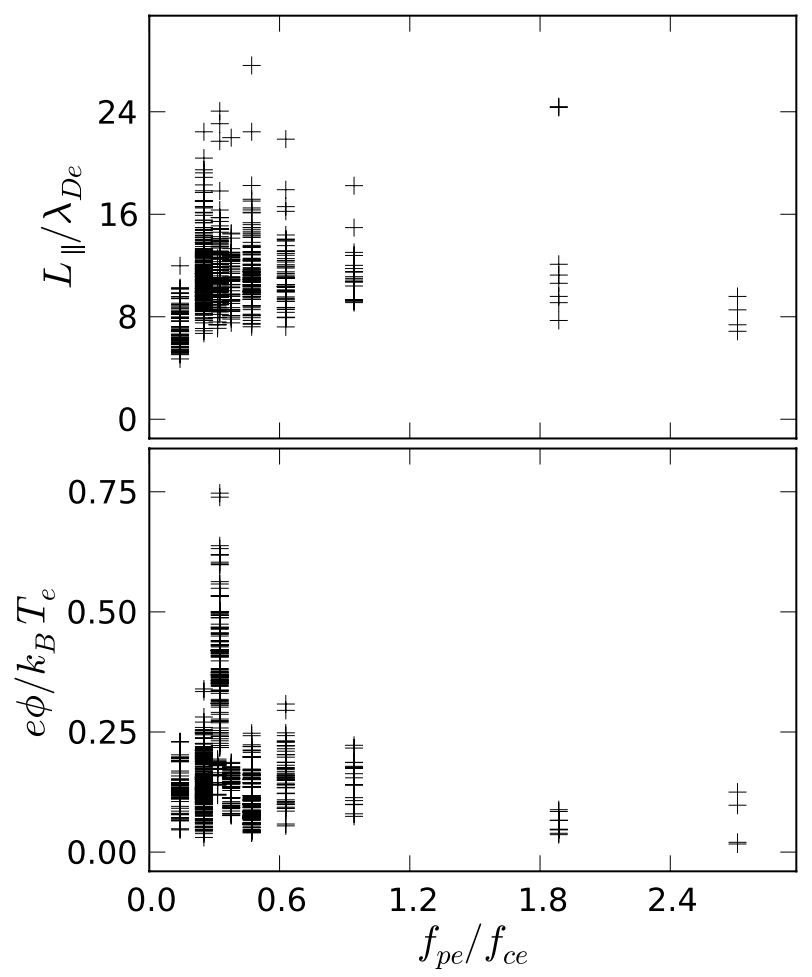

Fig. 8. Parallel half-width and amplitude as a function of $f_{\mathrm{pe}} / f_{\text {ce }}$ in the various experiments.

involving the beam (Lefebvre et al., 2010). On the other hand, the amplitudes reach larger values for larger beam voltages (Fig. 7). This is not very surprising however, since the beam is ultimately the free-energy source in the system from which fluctuations grow. But the solitary structures have to be a secondary product of the instability or be generated by a non-resonant mechanism.

Besides the beam voltage, other important parameters are the magnetic field strength and the plasma density. Figure 8 shows a broad range of widths and amplitudes at any given value of $f_{\mathrm{pe}} / f_{\mathrm{ce}}$, and tendency for the average widths and amplitudes to slightly decrease when this ratio increases although fewer experiments were conducted with $f_{\mathrm{pe}} / f_{\mathrm{ce}}>1$. Similar conclusions were found by Pickett et al. (2004a) in their survey of the structure properties in various places of the Earth's magnetosphere. Franz et al. (2000) found that $f_{\text {pe }} / f_{\text {ce }}$ is an important factor controlling the aspect ratio $L_{\|} / L_{\perp}$ of the structures (see also Berthomier et al., 2003). Detailed experimental studies of the perpendicular electric fields are left for future work.

\section{Discussion}

Solitary waves with a positive electric potential pulse detected after the injection of a suprathermal electron beam have half-widths ranging from 4.5 to $24.4 \lambda_{\mathrm{De}}$, amplitudes from 0.02 to $0.75 T_{\mathrm{e}} / e$ and velocities from 1.5 to $2.5 v_{\mathrm{Te}}$. The

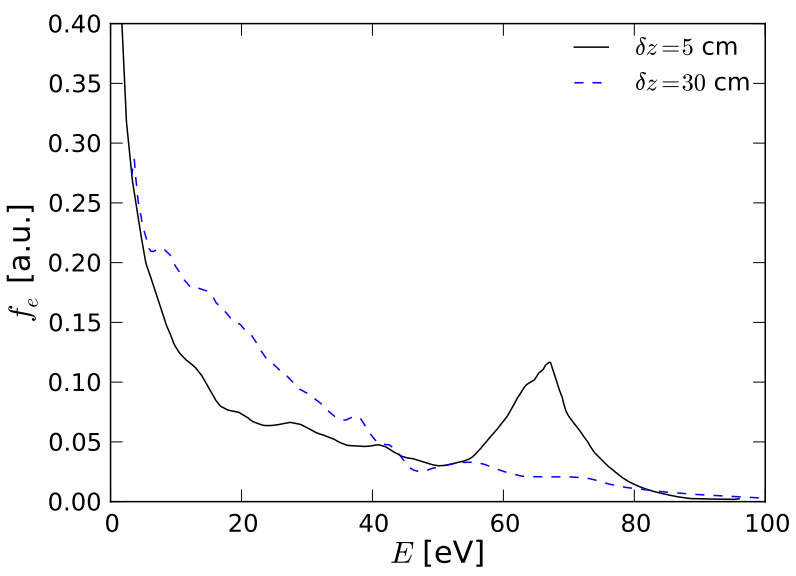

Fig. 9. Suprathermal part of the electron distribution function measured using a swept Langmuir probe 5 and $30 \mathrm{~cm}$ away from the beam source in the direction of the background magnetic field.

majority of them display a single potential pulse and dipolar electric field of the type shown on Fig. 2, corresponding to a positive charge density perturbation at the center of the structure (Lefebvre et al., 2010). The shape and scales of the solitary waves are consistent with electron holes. Furthermore, their amplitude and width satisfy an inequality for BGK-type electron holes derived by Chen et al. (2005).

Besides solitary pulses, waves and large amplitude wavepackets in a broad range of frequencies below $f_{\text {pe }}$ were detected. These fluctuations can be interpreted as quasielectrostatic whistler mode waves near the resonance, and the solitary pulses themselves were found to appear on the high frequency end of the dispersion curve (Lefebvre et al., 2010, Fig. 4). Since electron holes shaped as phasespace tubes have been found to decay into electrostatic whistlers (Goldman et al., 1999; Oppenheim et al., 1999), it could be suggested that the holes are the source of the waves. However in the present experiment the waves and wavepackets contain more power than the holes and are observed even in their absence, so it is more likely that a different generation mechanism is at work.

Essential information about the nature and origin of the waves and structures can be given by the electron velocity distributions. The suprathermal part of the electron distribution function was measured using a swept Langmuir probe capable of $100 \mathrm{~V}$ sweeps by taking the derivative of the I-V characteristic curve. Measurements were made 5 and $30 \mathrm{~cm}$ away from the $66 \mathrm{~V}$ beam source (Fig. 9). $5 \mathrm{~cm}$ away from the source along the background magnetic field $(\delta z=5$ $\mathrm{cm})$ measurements show a $62 \mathrm{eV}$ beam with temperature $T_{\mathrm{b}} \approx 5 \mathrm{eV}$ and a density of approximately $25 \%$ of the total density. An energetic tail on the background distribution extending up to the beam energy is also observed with a $30 \%$ relative density. At $\delta z=30 \mathrm{~cm}$ no trace of the beam is left and only a $35 \mathrm{eV}$ tail remains, a likely result of the scattering of the beam electrons by the waves and solitary structures. 
Most of the solitary structures were detected when the probes were put approximately $6 \mathrm{~cm}$ away from the beam source rather than at larger distances (no measurements were made at shorter distances in order to preserve the probes from melting due to beam electron bombardment). At this distance the beam is still clearly present. However because the waves and solitary structures have a much smaller velocity than the beam velocity (by approximately a factor 10), a resonant excitation mechanism by the beam electrons can be excluded. The measured velocities also exclude generation by a twostream instability. A more likely generation mechanism is instead a lower-hybrid instability driven by parallel currents similar to the one described by McMillan and Cairns (2006) (see also the discussion by Lefebvre et al., 2010).

Finally, let us note that an alternative interpretation of the solitary structures might be possible in terms of electron acoustic solitary waves. Indeed the measured electron distribution functions may support the type of electron acoustic solitary waves with a positive potential hump described by Berthomier et al. (2000). However the velocities of the structures are inconsistent with beamgenerated electron acoustic waves. By assuming the presence of two counter-streaming electron beams in addition to a core background population, Lakhina et al. (2009) derived a type of electron acoustic solitary structures which can have properties comparable to those of the structures we observe. However a single electron beam is present in our experiments. We therefore favor an interpretation in terms of electron phase-space holes.

\section{Conclusions}

We have studied in a laboratory experiment Debye-scale solitary structures with a positive electric potential pulse generated by a suprathermal electron beam. The shape, size and amplitude of the structures are similar to those observed in space plasmas and consistent with electron holes. Parallel velocity measurements and their absence of dependence on the beam voltage exclude a resonant excitation mechanism by the beam or a two-stream instability. Instead the electron holes are conjectured to be driven by the parallel currents associated to the electron beam.

Acknowledgements. The work at UNH was supported by DOE under grant DE-FG02-07ER54941, at Cornell by DOE DE-FG0207ER54942, and at UI by DOE grant DE-FG02-07ER54943 as well as by NASA Goddard Space Flight Center through NNX07AI24G. The Work at UCLA was done at the Basic Plasma Science Facility, BaPSF, which is supported by the NSF PHY-0531621 and DOE DE-FC02-07ER54918.

Edited by: B. Tsurutani

Reviewed by: R. P. Pottelette and G. S. Lakhina

\section{References}

Andersson, L., Ergun, R. E., Tao, J., Roux, A., Lecontel, O., Angelopoulos, V., Bonnell, J., McFadden, J. P., Larson, D. E., Eriksson, S., Johansson, T., Cully, C. M., Newman, D. N., Goldman, M. V., Glassmeier, K., and Baumjohann, W.: New Features of Electron Phase Space Holes Observed by the THEMIS Mission, Phys. Rev. Lett., 102, 225004, doi:10.1103/ PhysRevLett.102.225004, 2009.

Bale, S. D., Hull, A., Larson, D. E., Lin, R. P., Muschietti, L., Kellogg, P. J., Goetz, K., and Monson, S. J.: Electrostatic Turbulence and Debye-Scale Structures Associated with Electron Thermalization at Collisionless Shocks, Astrophys. J. Lett., 575, L25-L28, doi:10.1086/342609, 2002.

Berk, H. L., Nielsen, C. E., and Roberts, K. V.: Phase Space Hydrodynamics of Equivalent Nonlinear Systems: Experimental and Computational Observations, Phys. Fluids, 13, 980-995, doi:10.1063/1.1693039, 1970.

Berthomier, M., Pottelette, R., Malingre, M., and Khotyaintsev, Y.: Electron-acoustic solitons in an electron-beam plasma system, Phys. Plasmas, 7, 2987-2994, doi:10.1063/1.874150, 2000.

Berthomier, M., Pottelette, R., Muschietti, L., Roth, I., and Carlson, C. W.: Scaling of 3D solitary waves observed by FAST and POLAR, Geophys. Res. Lett., 30(22), 2148, doi: 10.1029/2003GL018491, 2003.

Bostrom, R., Gustafsson, G., Holback, B., Holmgren, G., and Koskinen, H.: Characteristics of solitary waves and weak double layers in the magnetospheric plasma, Phys. Rev. Lett., 61, 82-85, doi:10.1103/PhysRevLett.61.82, 1988.

Cattell, C., Crumley, J., Dombeck, J., Wygant, J. R., and Mozer, F. S.: Polar observations of solitary waves at the Earth's magnetopause, Geophys. Res. Lett., 29(5), 1065, doi:10.1029/ 2001GL014046, 2002.

Cattell, C., Dombeck, J., Wygant, J., Drake, J. F., Swisdak, M., Goldstein, M. L., Keith, W., Fazakerley, A., André, M., Lucek, E., and Balogh, A.: Cluster observations of electron-holes in association with magnetotail reconnection and comparison to simulations, J. Geophys. Res.-Space, 110, A01211, doi:10.1029/ 2004JA010519, 2005.

Chen, L.-J., Pickett, J., Kintner, P., Franz, J., and Gurnett, D.: On the width-amplitude inequality of electron phase space holes, J. Geophys. Res.-Space, 110, A09211, doi:10.1029/ 2005JA011087, 2005.

Chiang, F.: Micromachined probes for Laboratory Plasmas, Ph.D. thesis, University of California, 2009, IEEE T. Plasma. Sci., submitted, 2010.

Drake, J. F., Swisdak, M., Cattell, C., Shay, M. A., Rogers, B. N., and Zeiler, A.: Formation of electron-holes and Particle Energization During Magnetic Reconnection, Science, 299, 873877, doi:10.1126/science.1080333, 2003.

Ergun, R. E., Carlson, C. W., McFadden, J. P., Mozer, F. S., Muschietti, L., Roth, I., and Strangeway, R. J.: Debye-Scale Plasma Structures Associated with Magnetic-Field-Aligned Electric Fields, Phys. Rev. Lett., 81, 826-829, doi:10.1103/ PhysRevLett.81.826, 1998.

Fox, W., Porkolab, M., Egedal, J., Katz, N., and Le, A.: Laboratory Observation of Electron Phase-Space Holes during Magnetic Reconnection, Phys. Rev. Lett., 101, 255003, doi:10.1103/ PhysRevLett.101.255003, 2008. 
Franz, J. R., Kintner, P. M., Seyler, C. E., Pickett, J. S., and Scudder, J. D.: On the perpendicular scale of electron phase-space holes, Geophys. Res. Lett., 27, 169-172, doi:10.1029/1999GL010733, 2000.

Franz, J. R., Kintner, P. M., Pickett, J. S., and Chen, L.J.: Properties of small-amplitude electron phase-space holes observed by Polar, J. Geophys. Res.-Space, 110, A09212, doi: 10.1029/2005JA011095, 2005.

Gekelman, W., Pfister, H., Lucky, Z., Bamber, J., Leneman, D., and Maggs, J.: Design, construction, and properties of the large plasma research device - The LAPD at UCLA, Rev. Sci. Instrum., 62, 2875-2883, doi:10.1063/1.1142175, 1991.

Goldman, M. V., Oppenheim, M. M., and Newman, D. L.: Nonlinear two-stream instabilities as an explanation for auroral bipolar wave structures, Geophys. Res. Lett., 26, 1821-1824, doi:10.1029/1999GL900435, 1999.

Goldman, M. V., Newman, D. L., and Pritchett, P.: Vlasov simulations of electron-holes driven by particle distributions from PIC reconnection simulations with a guide field, Geophys. Res. Lett., 35, 22109, doi:10.1029/2008GL035608, 2008.

Guio, P., Børve, S., Daldorff, L. K. S., Lynov, J. P., Michelsen, P., Pécseli, H. L., Juul Rasmussen, J., Saeki, K., and Trulsen, J.: Phase space vortices in collisionless plasmas, Nonlin. Processes Geophys., 10, 75-86, doi:10.5194/npg-10-75-2003, 2003.

Lakhina, G. S., Singh, S. V., Kakad, A. P., Goldstein, M. L., Viñas, A. F., and Pickett, J. S.: A mechanism for electrostatic solitary structures in the Earth's magnetosheath, J. Geophys. Res.-Space, 114, 9212, doi:10.1029/2009JA014306, 2009.

Lefebvre, B., Chen, L.-J., Gekelman, W., Kintner, P., Pickett, J., Pribyl, P., Vincena, S., Chiang, F., and Judy, J.: Laboratory Measurements of Electrostatic Solitary Structures Generated by Beam Injection, Phys. Rev. Lett., 105, 115001, doi:10.1103/ PhysRevLett.105.115001, 2010.

Lynov, J. P., Michelsen, P., Pecseli, H. L., Juul Rasmussen, J., Saeki, K., and Turikov, V. A.: Observations of solitary structures in a magnetized, plasma loaded waveguide, Phys. Scripta, 20, 328335, doi:10.1088/0031-8949/20/3-4/005, 1979.

Mangeney, A., Salem, C., Lacombe, C., Bougeret, J.-L., Perche, C., Manning, R., Kellogg, P. J., Goetz, K., Monson, S. J., and Bosqued, J.-M.: WIND observations of coherent electrostatic waves in the solar wind, Ann. Geophys., 17, 307320, doi:10.1007/s00585-999-0307-y, 1999.

Matsumoto, H., Kojima, H., Miyatake, T., Omura, Y., Okada, M., Nagano, I., and Tsutsui, M.: Electrotastic Solitary Waves (ESW) in the magnetotail: BEN wave forms observed by GEOTAIL, Geophys. Res. Lett., 21, 2915-2918, doi:10.1029/94GL01284, 1994.

Matsumoto, H., Deng, X. H., Kojima, H., and Anderson, R. R.: Observation of Electrostatic Solitary Waves associated with reconnection on the dayside magnetopause boundary, Geophys. Res. Lett., 30, 1326, doi:10.1029/2002GL016319, 2003.

McMillan, B. F. and Cairns, I. H.: Lower hybrid turbulence driven by parallel currents and associated electron energization, Phys. Plasmas, 13, 052104, doi:10.1063/1.2198212, 2006.
Miyake, T., Omura, Y., Matsumoto, H., and Kojima, H.: Twodimensional computer simulations of electrostatic solitary waves observed by Geotail spacecraft, J. Geophys. Res., 103, 1184111850, doi:10.1029/98JA00760, 1998.

Morse, R. L. and Nielson, C. W.: One-, Two-, and ThreeDimensional Numerical Simulation of Two-Beam Plasmas, Phys. Rev. Lett., 23, 1087-1090, doi:10.1103/PhysRevLett.23. 1087, 1969.

Muschietti, L., Ergun, R. E., Roth, I., and Carlson, C. W.: Phasespace electron-holes along magnetic field lines, Geophys. Res. Lett., 26, 1093-1096, doi:10.1029/1999GL900207, 1999.

Oppenheim, M., Newman, D. L., and Goldman, M. V.: Evolution of Electron Phase-Space Holes in a 2D Magnetized Plasma, Phys. Rev. Lett., 83, 2344-2347, doi:10.1103/PhysRevLett.83.2344, 1999.

Pickett, J. S., Menietti, J. D., Gurnett, D. A., Tsurutani, B., Kintner, P. M., Klatt, E., and Balogh, A.: Solitary potential structures observed in the magnetosheath by the Cluster spacecraft, Nonlin. Processes Geophys., 10, 3-11, doi:10.5194/npg-10-3-2003, 2003.

Pickett, J. S., Chen, L.-J., Kahler, S. W., Santolk, O., Gurnett, D. A., Tsurutani, B. T., and Balogh, A.: Isolated electrostatic structures observed throughout the Cluster orbit: relationship to magnetic field strength, Ann. Geophys., 22, 2515-2523, doi:10.5194/angeo-22-2515-2004, 2004a.

Pickett, J. S., Kahler, S. W., Chen, L.-J., Huff, R. L., Santolk, O., Khotyaintsev, Y., Décréau, P. M. E., Winningham, D., Frahm, R., Goldstein, M. L., Lakhina, G. S., Tsurutani, B. T., Lavraud, B., Gurnett, D. A., André, M., Fazakerley, A., Balogh, A., and Rème, H.: Solitary waves observed in the auroral zone: the Cluster multi-spacecraft perspective, Nonlin. Processes Geophys., 11, 183-196, doi:10.5194/npg-11-183-2004, 2004b.

Pottelette, R. and Treumann, R. A.: Electron-holes in the auroral upward current region, Geophys. Res. Lett., 32, L12104, doi: 10.1029/2005GL022547, 2005.

Pritchett, P. L.: Onset and saturation of guide-field magnetic reconnection, Phys. Plasmas, 12, 062301, doi:10.1063/1. 1914309, 2005.

Roberts, K. V. and Berk, H. L.: Nonlinear Evolution of a TwoStream Instability, Phys. Rev. Lett., 19, 297-300, doi:10.1103/ PhysRevLett.19.297, 1967.

Saeki, K., Michelsen, P., Pécseli, H. L., and Rasmussen, J. J.: Formation and Coalescence of Electron Solitary Holes, Phys. Rev. Lett., 42, 501-504, doi:10.1103/PhysRevLett.42.501, 1979.

Schamel, H.: Hole equilibria in Vlasov-Poisson systems: A challenge to wave theories of ideal plasmas, Phys. Plasmas, 7, 4831-4844, doi:10.1063/1.1316767, 2000.

Temerin, M., Cerny, K., Lotko, W., and Mozer, F. S.: Observations of double layers and solitary waves in the auroral plasma, Phys. Rev. Lett., 48, 1175-1179, doi:10.1103/PhysRevLett.48.1175, 1982.

Tsurutani, B. T., Lakhina, G. S., Ho, C. M., Arballo, J. K., Galvan, C., Boonsiriseth, A., Pickett, J. S., Gurnett, D. A., Peterson, W. K., and Thorne, R. M.: Broadband plasma waves observed in the polar cap boundary layer: Polar, J. Geophys. Res., 103, 17351-17366, doi:10.1029/97JA03063, 1998. 